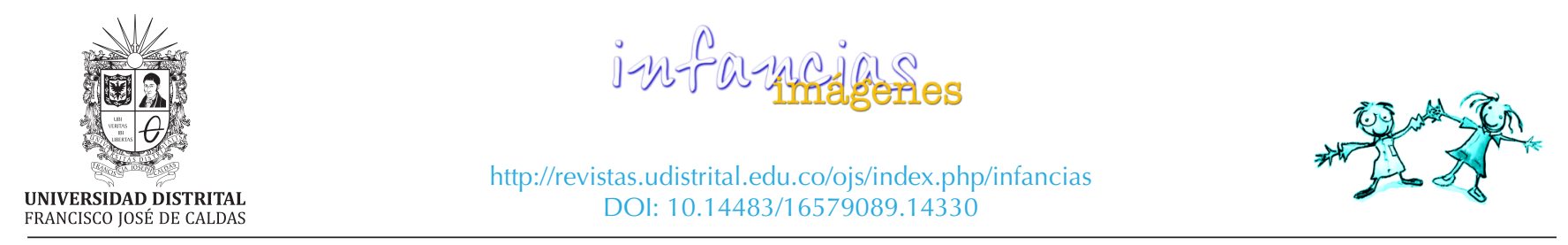

IMÁGENES DE INVESTIGACIÓN

\title{
El gusto por la escritura nace en el pensamiento*
}

\author{
The taste for writing is born in thought
}

\author{
Leonor Cortés Carrillo $^{1 \text { DD }}$, María Yaned Morales Benítez ${ }^{\text {(DD }}$
}

Para citar este artículo: Cortés, L., Morales, M. Y. (2020).

Recibido: 04-02-2019 - Aceptado: 25-05-2020

El gusto por la escritura nace en el pensamiento. Infancias

Imágenes, 19(2), 36-48

\section{Resumen}

Este artículo es el resultado de la investigación titulada "El desarrollo del pensamiento lógico-verbal y la implementación de estrategias de enseñanza dentro del marco Enseñanza para la Comprensión", realizada en la Universidad de la Sabana. Se analizaron las prácticas pedagógicas de la docente relacionadas con la planeación, su implementación y las estrategias trabajadas en el aula. Dicho análisis se desarrolló a partir de tres categorías: prácticas de planeación, enseñanza para la comprensión (EpC) y habilidades de pensamiento. Se usó como metodología la investigación-acción ya que permite analizar las prácticas pedagógicas y buscar las estrategias necesarias para alcanzar el objetivo de la investigación, con un enfoque cualitativo y un alcance descriptivo. Este ejercicio de investigación permitió evidenciar dentro de las prácticas aspectos como la importancia del uso constante del vocabulario del pensamiento, lo que permite abrir espacios para (de forma consiente) visualizar el pensamiento.

Palabras clave: habilidades de pensamiento, planeación, prácticas pedagógicas, comprensión.

\section{Abstract}

This article is the result of the reflexive process of investigation "the development of the thinking logical-verbal and the and the using of strategies into the teaching for comprehension framework", done in the Sabana University. It was necessary to analyze teacher's pedagogical practices related to planning, implementation, and the School-room strategies used. Such analysis was made from three different categories: planning practices, teaching for the comprehension (TFC), and thinking skills. Three pedagogical units were planned and analyzed after their implementation, with the purpose of making relevant adjustments under the TFC framework. The methodology used was the action research, because it allows us to analyze the pedagogical practices and look for the strategies needed to accomplish the aim of this research taking into account the qualitative approach and its descriptive scope. This research exercise made it possible to demonstrate within practices aspects such as the importance of the constant use of vocabulary of thought, allowing to open spaces to, in a conscious way, visualize the thinking.

Keywords: thinking skills, planning, pedagogical practices, comprehension-understanding.

* Este artículo reflexivo es producto del proyecto de investigación "El desarrollo del pensamiento lógico-verbal y la implementación de estrategias de enseñanza dentro del marco Enseñanza para la Comprensión", desarrollada entre julio de 2016 y junio de 2018, para optar al título de magíster en Pedagogía de la Universidad de la Sabana.

1 Licenciada en Educación Infantil y Preescolar, especialista en Procesos Lecto-escriturales y magister en Pedagogía, Universidad de la Sábana. Docente de la Secretaría de Educación de Cundinamarca. Correo electrónico: lecorca14@gmail.com. Orcid: https://orcid.org/00000002-4087-8593

2 Licenciada en Español e Inglés, especialista en Pedagogía de la Comunicación y Medios Interactivos y magister en Pedagogía. Docente de la Secretaría de Educación de Bogotá. Docente de la Facultad de Educación de la Universidad de la Sabana. Correo electrónico: mariamorben@unisabana.edu.co. Orcid: https://orcid.org/0000-0003-4916-3647 
"La escritura es un proceso de altísima elaboración que hace el cerebro y no la mano" Guzmán, Verela y Jorge (2010)

\section{Importancia de la palabra}

"Una palabra es un microcosmos de conciencia humana" (Vygotsky, 1995, p. 206). La palabra es la que abre las puertas a un mundo de posibilidades, donde los límites no existen; menos aún para los niños, quienes muestran una gran capacidad de inventiva y creación. Esa misma palabra registrada en códigos por medio de la escritura es la que permite dejar huellas a través de la historia; transmitir costumbres, creencias y descubrimientos; pues reconocer la existencia de lo que es el ser humano garantiza la preservación de la cultura, así como el movilizar y evidenciar los aprendizajes. Es tarea de los docentes lograr que los educandos desarrollen la habilidad de escribir como herramienta que les permita transmitir e inmortalizar sus pensamientos y conocimientos.

El docente debe reconocer la importancia y adecuada utilización del código escrito, así como el desarrollo de las habilidades de lectura, las habilidades propias del pensamiento y el reconocimiento de las características socioculturales de cada uno de los pequeños. Pero son el maestro y la familia en conjunto quienes desempeñan un papel muy importante, sobre todo en la etapa inicial de escolaridad.

Romero et al. (2009) plantean que:

Al ser esta etapa tan crucial, los maestros encargados de estos años de educación deben cumplir con dos requisitos esenciales. El primero de ellos, está relacionado con la capacidad del profesor para establecer relaciones positivas o auténticas con los menores... El segundo, las prácticas de los maestros deben estar fundamentadas, además de su experiencia, en un conocimiento científicamente basado en aspectos como el desarrollo de los niños, el desarrollo del alfabetismo, las prácticas de alfabetismo evolutivamente apropiadas, las pedagogías propias para niños preescolares, la identificación y el manejo de niños y niñas con necesidades educativas especiales y la importancia de trabajar con las familias para que contribuyan al éxito académico de los menores. (p. 80)
En relación con lo anterior, un niño que se inicia en el proceso de alfabetización y adquisición del código escrito sentirá mayor confianza cuando su maestro cumpla con los dos requisitos planteados, pues podrá darle experiencias significativas, agradables y sencillas que le permitirán tener un acercamiento al conocimiento con menos dificultades. Pero, además de los requisitos del maestro, si desde su primer contexto social (es decir, la familia) se encuentra rodeado de un entorno letrado las oportunidades de desarrollar las habilidades comunicativas con mayor rapidez y fluidez se potencian, lo cual se verá reflejado a lo largo de toda su vida y no solo la escolar (laboral, social y emocional).

Ya sea dicho entorno letrado, la lectura de literatura (cuentos, historias) o las mismas conversaciones que la familia realiza dentro o fuera de casa se trata de actividades que permiten el desarrollo del lenguaje oral en el niño. Este se convierte en la "base para el aprendizaje del lenguaje escrito" (Romero et al., 2009, p. 44), denominado código escrito.

De acuerdo con lo anterior, el código escrito es el "[...] conjunto de conocimientos de gramática y de lengua [...]" (Cassany, 2007, p. 11). Este se va disponiendo cuando los niños formalizan su acceso a la educación, en el aula de clase, donde poco a poco se les brinda diferentes conceptos y estructuras, todas relacionadas con la escritura, tales como: la forma adecuada de escribir una u otra palabra, la estructura de las oraciones, los conceptos gramaticales, normas de ortografía, entre tantos más.

Pero como también lo plantea Cassany (2007), el conocimiento de dicho código no es el todo, no convierte a los estudiantes ni al docente en escritores expertos, ya que es necesario desarrollar un conjunto de estrategias que permitan avanzar hacia un buen desarrollo de la escritura. Además, los docentes deben hacer que los estudiantes tomen conciencia sobre la importancia de generar espacios que les permitan reflexionar sobre los procesos de aprendizaje y evolución de sus capacidades y habilidades propias para aprender. Es decir, es necesario que desarrollen un enfoque metacognitivo, considerado por Flavel "como el conocimiento que uno tiene acerca de los propios procesos y productos 
cognitivos o cualquier otro asunto relacionado con ellos" (1985, citado en Alvares, 2009, p. 8).

\section{El pensamiento y la escritura}

En las aulas de clases el docente debe dar las indicaciones claras al momento de realizar una actividad que permita a los estudiantes evidenciar los procesos cognitivos que están desarrollando. Pero, ¿cómo evidencio los procesos cognitivos?, o ¿cómo evidencio el pensamiento en los estudiantes? o ¿cómo los niños se vuelven conscientes de sus pensamientos y esto para qué les sirve? Estas preguntas, y otras más, están permanentemente rondando la mente del docente, si bien es importante que los niños desarrollen el gusto por leer y escribir, además, que desarrollen habilidades que permitan hacerlo de forma fluida y bien estructurada, también es significativo que el docente pueda enseñarles a pensar y a tomar conciencia sobre sus procesos de aprendizaje.

Continuando con la importancia de desarrollar la habilidad de escribir, es esencial tener buenos hábitos de lectura. El docente debe leerles a sus estudiantes si desea formar buenos escritores. Pero la lectura debe ser aquella que se recrea con pasión, gusto e interés; aquella que utiliza diversos matices de voz cuando se realiza en voz alta, haciendo un adecuado uso de los signos paralingüísticos que permitan transportarse a esos palacios, ciudades encantadas o espacios estelares. "Lo paralingüístico en el lenguaje oral corresponde a los matices, los ritmos y las entonaciones de la voz que ayudan a resaltar la intención y el significado del mensaje" (Guzmán et al., 2010, p. 25).

La lectura realizada a los pequeños es la principal fuente de imaginación y apropiación de conocimientos y sueños de otros. Esta brinda un sin número de palabras al tiempo que alimenta el vocabulario y afianza el código escrito cuando se toma la decisión de escribir los propios sueños y plasmar el pensamiento.

Por otra parte, todo cuanto está escrito da razón de algo o de alguien; de individuos dentro de un pueblo o de una comunidad entera. Tal como lo plantea Vygotsky (1995), el desarrollo de los niños está íntimamente ligado a las interacciones comunicativas y sociales. Cuando se permite que los niños dentro y fuera del aula interactúen, dialoguen, intercambien conceptos para construir conocimientos y aprendizajes todo con un fin comunicativo y luego se les anima a registrar sus experiencias, los procesos de escritura serán más placenteros y menos impuestos. Lo que permite que poco a poco las destrezas y procesos de composición sean más fluidos. En este sentido, la escritura "es un proceso susceptible de ser enriquecido a lo largo de toda la vida" (Guzmán et al., 2010, p. 44).

Además, como docentes facilitadores de los procesos de escritura hay que dar a los niños las pautas necesarias para realizar un buen escrito. Que comprendan desde pequeños que la escritura se puede mejorar cuando se tiene claro cuál es el propósito al escribir, quienes leerán sus escritos y cómo deben organizar sus ideas.

Siguiendo en el mismo orden, es necesario que los estudiantes comprendan que no se logrará una versión definitiva con el primer escrito; que es preciso redactar un borrador, revisarlo, corregirlo una y otra vez, volverlo a leer, a revisar y, finalmente, a reescribirlo. Para ello se requieren unas fases: planificación, textualización, relectura, ajuste y, por último, la edición (Guzmán et al., 2010, pp. 47 y 48).

Según lo anterior, el lenguaje oral o escrito moviliza el pensamiento. Pero, ¿qué significa movilizar el pensamiento? Antes de responder esta pregunta es necesario hablar sobre el lenguaje del pensamiento: "las palabras enseñan conceptos y crean así senderos para que el pensamiento los recorra" (Thishman et al., 2006, p. 22). Si las palabras moldean y modifican el pensamiento, los docentes deben apropiarse del vocabulario adecuado y permitir que los niños accedan a este con el fin de mejorar los razonamientos, las comprensiones y evidenciarlos en las producciones textuales. Thishman et al. (2006, p. 26) nos proporcionan una serie de palabras o vocabulario de lenguaje de pensamiento que podemos utilizar en las aulas. A continuación, se dan algunos ejemplos: 
Tabla 1. Vocabulario del pensamiento

\begin{tabular}{|ccccc|}
\hline aprender & afirmar & analizar & Comprender & concluir \\
cuestionar & calcular & Deducir & definir & demostrar \\
evaluar & especular & inferir & investigar & justificar \\
meditar & negar & observar & pensar & planear \\
razonar & revisar & saber & suponer & verficiar \\
\hline
\end{tabular}

Fuente: adaptada de Thishman et al. (2006, p. 26).

Tabla 2. Movimientos de pensamiento

\begin{tabular}{|ll|}
\hline 1. & Observar de cerca y describir que hay ahí. \\
2. & Construir explicaciones e interpretaciones. \\
3. & Razonar con evidencia. \\
4. Establecer conexiones. \\
5. Tener en cuenta diferentes puntos de vista y perspectiva. \\
6. Captar lo esencial y llegar a conclusiones. \\
7. Preguntarse y hacer preguntas. \\
8.
\end{tabular}

Fuente: Richhart et al. (2014, p. 46).

Los autores también formulan la pregunta ¿cómo ayuda el lenguaje de pensamiento a los alumnos a que piensen mejor? Nos dan dos razones que responden muy bien la pregunta: "ayuda a los alumnos a organizar y comunicar su propio pensamiento con mayor precisión e inteligencia; además, el lenguaje del pensamiento comunica y refuerza las normas del pensamiento" (Thishman et al., 2006, p. 27). Así mismo, como lo plantean Guzmán et al. (2010), en los referentes didácticos del lenguaje ciclo tres "el lenguaje es una poderosa herramienta que moviliza el pensamiento" (p. 17).

Con relación a los movimientos del pensamiento, es importante resaltar que mediante estos se puede acceder a la comprensión. Los investigadores del proyecto Zero de Harvard (Richhart et al., 2014) definieron algunos tipos de pensamiento que se deben trabajar en el aula con el fin de promover en los estudiantes las habilidades del pensamiento que les permiten más y mejores comprensiones, incrementan la capacidad de argumentar e interpretar (no solo lo que escriben o leen) y también mejoran las habilidades discursivas. La tabla 2 muestra dichos movimientos:
La comprensión es uno de los procesos cognitivos más complejos y depende de la calidad del pensamiento. Esta se va desarrollando y modificando, incluso "se podría decir que se perfecciona a lo largo de la vida" (Restrepo, 2017, p. 85) y permite la apropiación del conocimiento. Tal como lo afirman Richhart et al.: "en la medida en que los estudiantes son más conscientes de su propio pensamiento y de las estrategias y procesos que utilizan para pensar, se vuelven más metacognitivos" (2014, p. 47).

Así, se hace necesario llevar al aula de clase el vocabulario del pensamiento y permitir que los niños se familiaricen con este, lo utilicen, lo conozcan y luego se apropien del mismo. Cuando en el aula un niño no solo utiliza el léxico cotidiano propio de sus edades o de las áreas que está trabajando, sino que al expresarse utiliza términos como los expuestos en la tabla 1, poco a poco su conciencia de aprendizaje y el cómo logra ese aprendizaje será parte de su nueva forma de acceder al conocimiento. Luego, cuando se introducen los movimientos del pensamiento a la cotidianidad, los niños evidencian realmente cómo es que piensan y cuál es la mejor ruta para responder a las necesidades cotidianas de 
forma más acertada y consiente poniendo en relieve sus verdaderas comprensiones, lo que nos permite dar respuesta a la pregunta hecha sobre ¿qué es movilizar el pensamiento?

\section{El pensamiento en el aula}

¿Pero de dónde nace la necesidad de trabajar las habilidades del pensamiento y mejorar los procesos escriturales de los niños? Gracias a la investigación, al indagar cuál sería la situación problema en el aula de clase, se pudo evidenciar que los estudiantes de grado quinto, con edades entre los 9 a 12 años, de la escuela rural donde labora y a quienes supuestamente conocía muy bien (pues está con ellos desde hace cinco años), en realidad no estaban pensando de forma adecuada. Se encontró que no tenían la capacidad de sustentar, argumentar, defender o discrepar las ideas propias y las de sus compañeros ni mucho menos plasmarlas en sus textos.

Luego, con las lecturas de expertos, se logró revelar que los estudiantes podrían encontrarse en el primer nivel de pensamiento que plantea Villarini (2003): el automático. De modo que Ilevarlos al siguiente nivel implicaría modificar sus clases y sus planeaciones con el fin de potencializar realmente las habilidades del pensamiento. $Y$ este cambio debería verse reflejado en las intervenciones orales de sus estudiantes (sin importar el área de conocimiento o el lugar) y en las composiciones escritas (en tanto se prevé que estén mejor estructuradas y plasmen realmente los propios pensamientos).

¿Por qué se consideraba que los niños al comienzo de la investigación se encontraban en un nivel de pensamiento automático? La docente se percató de que la mayoría, por no decir que todos, respondían de forma automática. Como lo aclara Villarini "a veces actuamos 'sin pensarlo mucho', pensamos automáticamente, es decir, respondemos de modo inmediato ante los diversos estímulos del ambiente con respuestas previamente aprendidas" (2003, p. 38). al detenerse a observar las intervenciones de los estudiantes reconoció que los niños no se detenían a pensar, respondían por responder y muchas veces ellos mismos se daban cuenta de que sus respuestas no tenían sentido. Lo anterior se evidencia en las transcripciones de videos de clases, en diálogos o conversaciones en el aula, diarios de campo, incluso en registros fotográficos de trabajos de clase.

En la figura 1 se muestra una respuesta automática. Es decir, poco pensada al trabajo solicitado de completar dos frases con el fin de darle sentido al texto y mostrar los aprendizajes relacionados con un tema de matemáticas. Si bien la investigación está enfocada en potencializar el pensamiento lógico-verbal de la escritura, este pensamiento es transversal a todas las áreas de conocimiento.

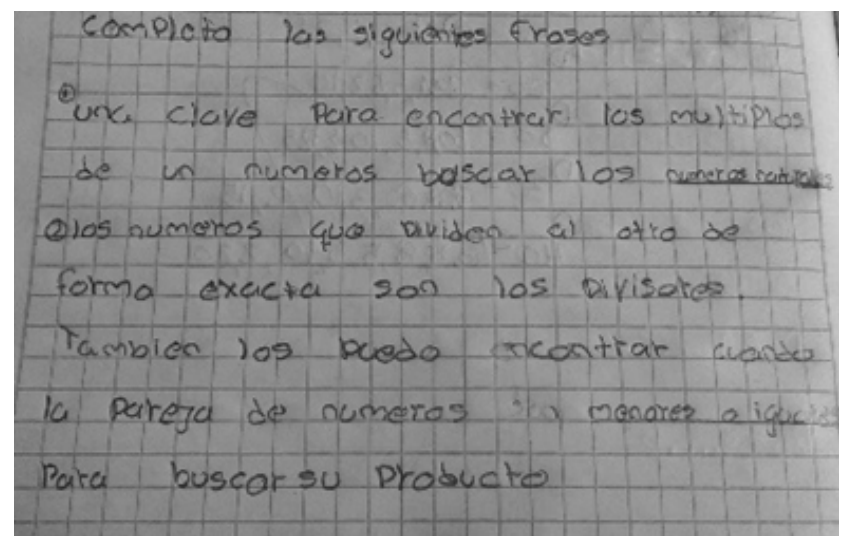

Figura 1. Texto elaborado por un estudiante de grado 5 de 10 años.

Ahora bien, la tarea era indagar sobre cómo la docente podía llevar a los niños a otro nivel de pensamiento. En este punto intervienen las nociones de pensamiento, habilidad, destreza, métodos y estrategias, estudiadas por los expertos y que se deben aterrizar al aula de clase para lograr el objetivo ya planteado para los niños fueran capaces de detenerse a pensar y se dieran el tiempo para hacerlo, así como el resolver problemas, tomar de forma adecuada cualquier decisión y comunicarse de forma efectiva. ViIlarini (2003) muestra que el proceso de pensamiento puede tener lugar en tres niveles de funcionamiento mental consciente, como se ve en la figura 2.

Por lo anterior, se hace necesario producir un cambio, pero no solo en los niños, sino en las prácticas pedagógicas y en las acciones desarrolladas en el aula. Un cambio centrado en mejorar y potencializar el desarrollo del pensamiento, ya que cuando el docente no propicia espacios adecuados para analizar, refutar, sustentar y desarrollar un pensamiento crítico la labor no está del todo cumplida; solo se convierte en transmisor de contenidos y el aprendizaje difícilmente se puede dar. Al no 
fomentar el pensamiento, sus funciones y productos tampoco se potencializan, lo que podría generar ciudadanos poco racionales, introvertidos, que presentan dificultades en la toma de decisiones y resolución de problemas.

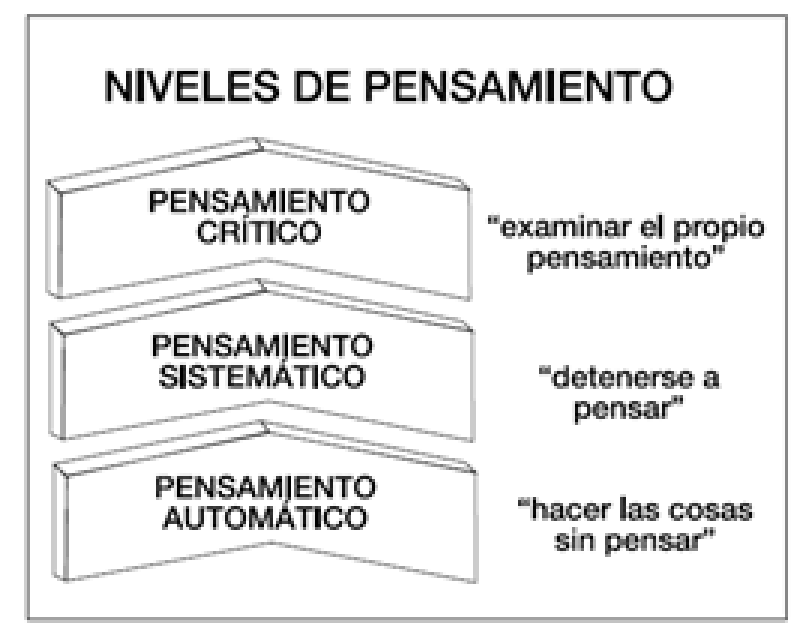

Figura 2. Niveles del pensamiento.

Fue.nte: Villarini (2003, p. 23).

Para Perkins y Vera "la enseñanza debe centrarse en el pensamiento, en donde impere la reflexión y el dinamismo" (citados en Ruiz, 2006, p 3). Esto permite que el estudiante sea consciente de sus procesos y aplique sus conocimientos de forma más efectiva para generar un verdadero aprendizaje.

En este sentido, es de gran importancia que en las aulas se propicien espacios para aprender a utilizar estrategias de pensamiento que permitan a los estudiantes apropiarse del conocimiento y aplicarlo adecuadamente, según sean las necesidades del entorno donde se desenvuelven. Como afirma Johnson: "Mientras que los conocimientos acumulados pueden cambiar, desdibujarse o tornarse inútiles, la capacidad de pensar de manera eficaz permanece constantemente. Las estrategias de pensamiento nos permiten adquirir el conocimiento necesario y aplicarlo adecuadamente" (2003, p. 11). El autor recalca la importancia que se le debe dar al pensamiento, más que a las temáticas o la acumulación de conocimiento sin sentido. Este es un Ilamado de atención a los docentes; a despertar del letargo en que se ha caído por el afán de cumplir con los planes establecidos y exigidos en las instituciones educativas, que a su vez están preocupadas por cumplir con las políticas educativas estatales.

A partir de los referentes teóricos, de las observaciones realizadas a las planeaciones y al desarrollo de las clases, la investigadora plantea las siguientes categorías de análisis con el fin de seleccionar, agrupar y clasificar aquellas acciones relacionadas con la investigación que tiene gran importancia para la misma. Esto, en consonancia con lo planteado por Hernández et al. (2006) cuando afirman que las categorías deben relacionarse lógicamente con los datos que representa. La figura 3 evidencia las categorías seleccionadas y sus subcategorías.

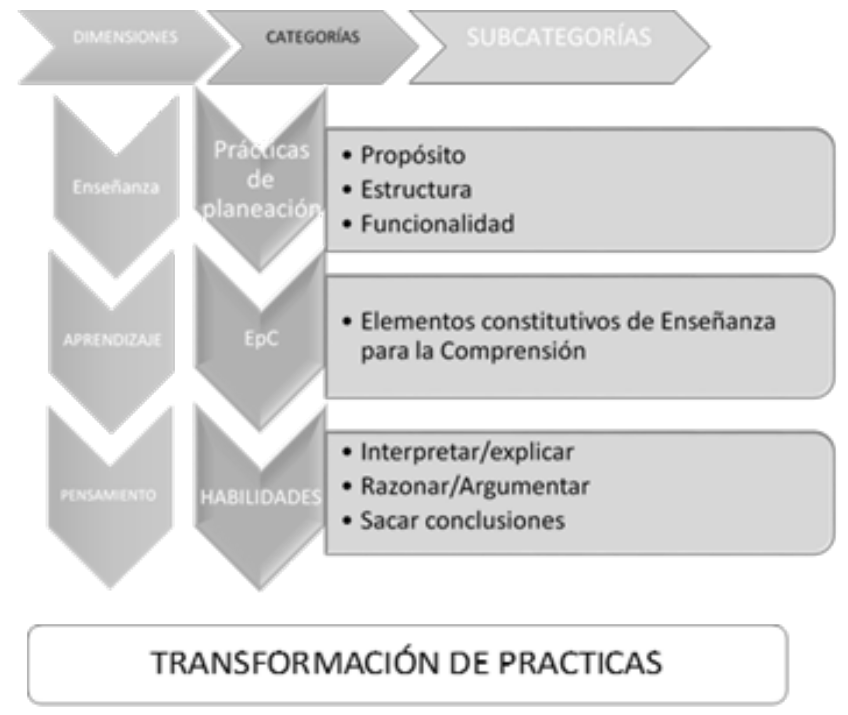

Figura 3. Categorías de análisis.

Fuente: elaboración propia.

\section{Definición de categorías}

\section{Planeación}

"El plan de clase es un instrumento teórico-metodológico que tenemos a nuestro alcance todos los profesores. Aprender y comprender la esencia de dicho instrumento permite a los educadores visualizar con antelación el camino viable para el logro de aprendizajes" (Rodríguez, 2009, p. 1). Este instrumento permite encaminar el proceso de enseñanza. Es necesario que todo docente tenga claro los componentes necesarios para realizar una planeación consiente, dedicarle el tiempo adecuado y evaluar si la planeación y desarrollo de la misma es efectiva y garantiza la adquisición de comprensiones. 


\section{Enseñanza para la comprensión}

"La EpC no es una solución sino una estructura para estimular y construir diálogos acerca de lo que debería aprenderse y cómo enseñarlo" (Stone, 1999, p. 432). Es una estrategia que orienta al docente para mejorar sus prácticas pedagógicas en pro de cumplir con el fin de la educación: formar personas autónomas y críticas capaces de confrontar diferentes circunstancias de la vida con éxito.

\section{Habilidades de pensamiento}

Es la capacidad de pensar de forma eficaz. Permite acercar al estudiante a la comprensión y la toma adecuada de decisiones, así como el desarrollar la capacidad argumentativa y ser más crítico y creativo (habilidades que le permitirán mejorar su proyecto de vida).

\section{Fuentes e instrumentos de recolección}

Se refiere la gran variedad de técnicas o herramientas que nos ayudan a recolectar información. Según Latorre:

Tenemos tres formas tradicionales de recoger información (datos) o tres maneras de averiguar lo que pasó. Podemos observar lo que las personas dicen o hacen y tratar de descubrir lo que ocurrió, o podemos preguntarles sobre lo que ocurrió, o también podemos analizar los materiales o huellas que dejaron. (2005, p. 54)

En este sentido, las técnicas utilizadas que apoyaron el proceso de investigación fueron:

1. Prueba Saber 2015: permite establecer posibles avances académicos.

2. Entrevistas: se realizaron a docentes, estudiantes y grupo interdisciplinario (profesora de inglés, fonoaudióloga y deportólogo) sobre la percepción del clima de aula y procesos académicos de los estudiantes de la sede y del grado.

3. Diarios de campo: permitieron registrar las percepciones del docente frente al desarrollo de la planeación de clases en relación con el desarroIlo del pensamiento lógico-verbal, la aceptación y eficacia de la misma.

4. Formato de planeación: con este instrumento se pretendió verificar la funcionalidad en el proceso de planeación, los aportes al desarrollo del pensamiento y la efectividad y claridad al momento de poner en práctica las unidades planeadas.

5. Audios y videos de clases: permiten observar aspectos que en el momento del desarrollo de la clase no son perceptibles, permitiendo reconocer posibles errores o falencias que se pueden enmendar en la siguiente planeación y desarroIlo de la clase, lo que favorece los procesos de enseñanza-aprendizaje.

6. Matrices de análisis de movimientos de pensamiento: al aplicar estas matrices a las unidades de EpC se pudo ver los niveles inicial, intermedio y avanzado de los movimientos de pensamiento: interpretar-explicar, razonar y argumentar y sacar conclusiones.

7. Matriz valorativa unidades de EpC: esta matriz fue aplicada a los proyectos finales de síntesis de tres unidades de clase: figuras literarias, siluetas textuales y los conectores nos conectan. Con el fin de verificar la comprensión y apropiación de los conceptos trabajados en cada unidad se establecieron tres criterios: básico, intermedio y avanzado.

8. Matriz niveles de pensamiento: este instrumento permitió establecer el nivel de pensamiento en el que se encontraban los estudiantes. Se aplicó a trabajos de los estudiantes, no solo en el área de español, sino también de matemáticas; en esta se establecieron los niveles de pensamiento que plantea Villarini (2003): automático, sistémico y analítico.

9. Matriz de planeación: permite tener claridad sobre los criterios de la planeación, así como evaluar el proceso de planeación en el aula y cómo la realización eficaz de la misma mejora los procesos de comprensión de los estudiantes y el desarrollo en general de las clases. Se aplicó a las tres unidades de clase: figuras literarias, siluetas textuales y los conectores nos conectan.

A pesar de que las tres dimensiones (enseñanza, aprendizaje y pensamiento) están estrechamente relacionadas, por las características ya mencionadas, el centro de atención está en la dimensión de enseñanza y, una vez fortalecida, las demás dimensiones también lo harán de forma automática. 
Se decidió intervenir y empezar por trabajar el lenguaje del pensamiento expuesto en la tabla 1, con la firme convicción de que este es el primer paso para desarrollar en el aula una cultura de pensamiento. Para esto se ubicó en el salón de clase una cartelera con el vocabulario para familiarizar a los estudiantes y, cada vez que se necesitó, reforzar o desarrollar alguna actividad que involucrará las habilidades del pensamiento. Dicho vocabulario se retomó de forma contextualizada, evidenciando que cuando el niño y el docente se familiarizan con estos y otros términos realmente se adquiere una mayor conciencia y deseos por aprender; además de reconocer aquellas deficiencias que dificultan dicho proceso.

\section{Transformando las prácticas pedagógicas dentro del marco de EpC} Fue necesario plantear cambios en la forma en que la docente acercaba a los estudiantes al conocimiento y el cómo preparaba sus planeaciones, tomando como referencia el marco de EpC. Pues este permite tanto a docentes como estudiantes indagar, profundizar y encontrarse con las formas personales de comprender; y, como lo plantea Stone, "nunca puede ser simplemente transmitida de un generador aun receptáculo, sino que debe ser construido a partir de la propia experiencia y del trabajo intelectual" (1999, p. 26).

En este sentido, se retoman los elementos propios de la EpC y se aplican a las planeaciones de clase, pues el método de planeación que se estaba utilizando no respondía a la necesidad de fortalecer y desarrollar el pensamiento lógico-verbal enfocado a la escritura.

\section{Proceso de transformación}

La planeación inicial utilizaba un formato en el que se limitaba solo a realizar una descripción de las actividades a desarrollar con el fin de cumplir con el plan temático según el área de conocimiento, como se muestra en la tabla 3.

Esto llevó a modificar la forma de planear las clases para evitar hacer una serie de actividades que, aunque puedan estar enlazadas, en algún momento pierden su propósito. Es necesario que la docente plantee un objetivo claro al momento de planear y permita tanto la evaluación como la retroalimentación.

Tabla 3. Fragmento de formato de planeación inicial

Docente: Leonor Cortés Carrillo

Grado: 501

Periodo: segundo trimestre

Fecha: julio 4 al 28

\begin{tabular}{|c|c|}
\hline Área Español & $\begin{array}{l}\text { DBA: Comprende un texto leído } \\
\text { Reconoce los elementos de la lírica, que refuerza el significado de poemas y caligramas. }\end{array}$ \\
\hline Metodología pedagógica. & Actividad y desarrollo de la clase. \\
\hline $\begin{array}{l}\text { Trabajo colaborativo, inter- } \\
\text { pretación y clasificación de } \\
\text { información. } \\
\text { Debate. Coevaluación y } \\
\text { heteroevaluación. } \\
\text { Trabajo grupal e individual. }\end{array}$ & $\begin{array}{l}\text { La docente retoma el texto "himno a los pájaros" haciendo especial énfasis en los versos que } \\
\text { rima; pide a los niños que definan que es rima para ellos; luego de llegar a un acuerdo los niños } \\
\text { registrarán sus conceptos en el cuaderno y darán algunos ejemplos tomados de la misma lectura. } \\
\text { La docente escribe en el tablero una serie de palabras, los niños deberán buscar otra palabra que } \\
\text { rime y luego hacer una oración con las mismas. La docente escribe el poema "El fantasmita" y } \\
\text { los niños deberán buscar el conjunto de palabras que riman. En casa los niños deberán buscar } \\
\text { algunas adivinanzas, coplas y refranes y buscar en ellos las palabras que riman. }\end{array}$ \\
\hline Observación. & $\begin{array}{l}\text { Se desarrolla las actividades en los diferentes días asignados para el tema. Los estudiantes son } \\
\text { receptivos y participan activamente, pero la mayoría de los niños presentan dificultad al momen- } \\
\text { to de definir las figuras literarias. Al comparar sus conceptos con los expuestos en los libros e } \\
\text { internet realizan las correcciones necesarias. La docente en algunos momentos siente que la clase } \\
\text { se torna desordenada por el constante hablar de los estudiantes, además se preocupa mucho por } \\
\text { el tiempo al creer que no alcanzara a terminar las actividades. La visita de la asesora realizada el } \\
\text { día del cierre del tema y el video de clase aporta grandes observaciones de aspectos que no se } \\
\text { tuvieron en cuenta y que en el futuro podrían mejorar el desempeño de las actividades. }\end{array}$ \\
\hline
\end{tabular}

Fuente: elaboración propia. 
Los conocimientos adquiridos sobre EpC y sus elementos permitieron la transformación del formato de planeación, como lo muestra la tabla 4. Pero, más allá de eso, se modificaron estructuras mentales en la docente, lo que permitió que el desarrollo de la clase fuera más fluido, pero ante todo participativa. Lo que permitió que los niños realmente actuaran como agentes partícipes en la construcción de conocimientos basados en la comprensión.

Tabla 4. Fragmento de planeación con el marco de EpC

\begin{tabular}{lll}
\hline Docente: Leonor Cortés Carrillo & Grado: 506 & Área: Español \\
\hline $\begin{array}{l}\text { DBA } \\
\text { - Consulta diversas fuentes, organiza y selecciona la }\end{array}$ & $\begin{array}{l}\text { Tema: ¿los textos tienen silue- } \\
\text { ta como los humanos? }\end{array}$ & $\begin{array}{l}\text { Recursos: textos (cuento, no- } \\
\text { ticia, receta), hojas blancas, } \\
\text { información a presentar. }\end{array}$ \\
\cline { 2 - 3 } - Comprende un texto leído. & Fecha: agosto 7 al 31. & vídeo, cuadernos, lápices.
\end{tabular}

\section{Metas de comprensión}

\section{Conocimiento}

Los estudiantes comprenderán que cada texto tiene una estructura o silueta textual.

\section{Comunicación}

Los estudiantes comprenderán que pueden comunicar sus ideas utilizando de forma adecuada la estructura de las tipologías textuales.

\section{Método}

Los estudiantes comprenderán que, siguiendo las estructuras de los diferentes textos, los pueden elaborar de forma adecuada.

\section{Propósito}

Los estudiantes comprenderán que Hay diferentes formas de comunicarnos y es importante reconocer las tipologías textuales.

\begin{tabular}{llll}
\hline MC & Desempeños de comprensión & TD & Valoración continua \\
\hline
\end{tabular}

\section{Retroalimentación}

\section{Observación de los estudiantes}

En relación con el desempeño uno, los niños se mostraron muy receptivos y a gusto con las lecturas, pero al interior de los grupos se presentaron algunas dificultades para ponerse de acuerdo al momento de diseñar las posibles estructuras.

Una vez dada la explicación los niños presentaron sentimientos encontrados al darse cuenta de que su observación y selección al escribir las partes de su estructura eran acertadas y otras no.

\section{Observación de las prácticas pedagógicas}

Es necesario anticipar estrategias que permitan la escucha activa al interior de los grupos, pues la intención de sobreponer sus ideas provoca que algunos estudiantes no escuchen las ideas de los compañeros.

Es conveniente tener más lecturas que sirvan de ejemplo para cada tipología textual.

1 y 2 1. Los estudiantes, recibirán tres textos cortos:

3 y 4 narrativo, instructivo e informativo. Leerán los textos y escribirán las principales características de cada uno. Luego compararán con un compañero las características señaladas, explicando cada una. Seguido se organizarán en grupos de tres integrantes, discutirán las características y realizarán un (posible) esquema que contenga la estructura que debe tener cada texto guiándose con las características anteriormente discutidas.

Los estudiantes observaran la presentación sobre las siluetas textuales y las características comunicativas propias de cada tipología. Se dará un espacio para la discusión sobre las partes que debe tener cada silueta, su importancia dentro del mismo y la consecuencia de omitir uno o varios de las mismas. Leerán nuevamente cada texto y discutirán sobre su intención o propósito comunicativo.
Se plantearán de común acuerdo con los estudiantes.

\section{IG Criterios}

Lectura silenciosa de cada texto.

Lista de características de cada texto.

Orden y participación activa al interior de cada grupo.

Entrega de posible estructura de cada tipología textual.

\section{Retroalimentación}

Acompañamiento informal permanente por parte de la docente al interior de cada grupo aclarando posibles dudas.

\section{Criterios}

Atención a la presentación de las siluetas textuales, participación activa y respetuosa.

\section{Retroalimentación}

El docente de manera informal dará respuesta a preguntas y promoverá la generación de nuevas inquietudes.

Fuente: elaboración propia. 
Luego de aplicar y analizar la nueva planeación bajo los criterios de EpC, se evidenció que aún no respondía a la necesidad de trabajar las habilidades del pensamiento, por lo que se le hizo una nueva modificación y se incorporaron los movimientos del pensamiento. En la tabla 5 se muestra la nueva forma de preparación de clases. Además, se elaboraron carteleras con el fin de mostrar a los estudiantes los movimientos del pensamiento que se trabajarían en la unidad.

La puesta en acción de la nueva forma de planear, preparar y presentar las clases fue bien recibida por los niños; los cuales mostraron adquirir conciencia de la importancia del pensamiento y de su adecuada utilización en los procesos comunicativos en la apropiación del nuevo lenguaje del pensamiento.

Tabla 5. Fragmento de planeación de clase con EpC y movimientos de pensamiento

\begin{tabular}{|c|c|c|}
\hline Docente: Leonor Cortés Carrillo & Grado: 506 & Área: Español \\
\hline \multirow{2}{*}{$\begin{array}{l}\text { DBA } \\
\text { - Usa conectores de continuidad, condición, oposición y } \\
\text { orden para dar coherencia al texto. } \\
\text { - Comprende un texto leído }\end{array}$} & $\begin{array}{l}\text { Tema: conectores } \\
\text { Hilo conductor: los co- } \\
\text { nectores nos conectan. }\end{array}$ & \multirow{2}{*}{$\begin{array}{l}\text { Recursos: libro Lenguaje en- } \\
\text { tre textos 5, diapositivas, pro- } \\
\text { yector de video, computador, } \\
\text { video https://www.youtube. } \\
\text { com/watch?v=0yK64bAjrOw, } \\
\text { pliegos de papel periódico } \\
\text { con rutina de pensamiento } \\
\text { guías. }\end{array}$} \\
\hline & Fecha: septiembre. & \\
\hline
\end{tabular}

\section{Movimientos del} pensamiento

\begin{tabular}{lll} 
Interpretar/explicar & Razonar/Argumentar & Sacar conclusiones \\
\hline Al escuchar y participar & Al justificar el uso de & Al definir el concepto. \\
$\begin{array}{l}\text { en la lectura y seleccio- } \\
\text { nar las palabras. }\end{array}$ & los conectores en la & \\
\hline
\end{tabular}

Metas de comprensión

\section{Conocimiento}

Los estudiantes comprenderán que el uso de conectores permite dar coherencia al texto. Además, que se debe seguir un procedimiento estratégico para su elaboración.

\section{Comunicación}

Los estudiantes comprenderán que pueden comunicar sus ideas utilizando de forma adecuada los elementos estructurales de un texto.

\section{Propósito \\ Los estudiantes comprende- rán que al escribir un texto se deben tener en cuenta algunos elementos relacio- nados con su estructura y contenido.}

\section{Método}

Los estudiantes comprenderán que siguiendo el uso adecuado de los conectores y el procedimiento estratégico pueden producir una primera versión de texto con coherencia y cohesión, y luego hacerle las correcciones necesarias para una segunda versión.

\begin{tabular}{|c|c|c|c|}
\hline MC & Desempeños de comprensión & TD & Valoración continua \\
\hline 1 y 2 & $\begin{array}{l}\text { La docente solicitará a los niños que realicen } \\
\text { lectura "Amor y amistad, una fecha para cele- } \\
\text { brar" del libro Lenguaje entre textos 5. }{ }^{\circ} \text {, luego } \\
\text { la docente solicitará a un niño que realice } \\
\text { nuevamente la lectura en voz alta. La docente } \\
\text { realizará una serie de preguntas para verificar } \\
\text { la comprensión de la lectura. Algunas pueden } \\
\text { ser: ¿cuándo celebramos en Colombia el día del } \\
\text { amor y la amistad?, ¿qué particularidades tiene } \\
\text { esta fecha?, ¿estás de acuerdo con esta cele- } \\
\text { bración?, ¿qué añadirías? Se dejará suficiente } \\
\text { tiempo para participar en el conversatorio. }\end{array}$ & $E$ & $\begin{array}{l}\text { Se plantearán de común acuerdo } \\
\text { con los estudiantes. } \\
\text { Criterios: lectura silenciosa del } \\
\text { texto, escucha activa, atención y } \\
\text { participación. } \\
\text { Retroalimentación. } \\
\text { Acompañamiento informal perma- } \\
\text { nente por parte de la docente. }\end{array}$ \\
\hline
\end{tabular}


Retroalimentación

\begin{tabular}{ll}
\hline Observación de los estudiantes & Observación de las propias prácticas \\
\hline Los niños en sus grupos de trabajo participaron en la rutina PIE & $\begin{array}{l}\text { Evidentemente, cuando se tiene claro lo que los } \\
\text { y se evidenció que muchos tenían idea de qué son los conec- } \\
\text { niños deben realizar y el cómo la clase se hace más }\end{array}$ \\
$\begin{array}{l}\text { video y curiosidad al ver las diferentes clases de conectores que } \\
\text { existen. Esta vez Kevin y Michael hacían una y otra vez pregun- }\end{array}$ & $\begin{array}{l}\text { a través de los movinos podían evidenciar sus pensamientos fue muy bueno, pues } \\
\text { tas como: "¿el uso inadecuado de los conectores es lo que hace } \\
\text { que el texto no tenga sentido?, ¿qué pasa si en vez de utilizar }\end{array}$ \\
uno u otro conector los mezclo entre sí?". & \\
\hline Anexos &
\end{tabular}

Fuente: elaboración propia.

Tabla 6. Matriz Movimientos del pensamiento

\begin{tabular}{|c|c|c|c|}
\hline Movimiento del pensamiento & Nivel inicial & Nivel intermedio & Nivel avanzado \\
\hline Interpretar/explicar & $\begin{array}{l}\text { Elabora explicaciones } \\
\text { con poco sentido, } \\
\text { evidenciando su bajo } \\
\text { nivel de interpretación. }\end{array}$ & $\begin{array}{l}\text { Explica la información } \\
\text { recibida según sus propias } \\
\text { interpretaciones sin tener } \\
\text { en cuenta en su totalidad la } \\
\text { información recibida. }\end{array}$ & $\begin{array}{l}\text { Establece relaciones con } \\
\text { referencia a la información } \\
\text { recibida y realiza explicacio- } \\
\text { nes con sentido. }\end{array}$ \\
\hline Razonar/argumentar & $\begin{array}{l}\text { Sus intervenciones ca- } \\
\text { recen de argumentos. }\end{array}$ & $\begin{array}{l}\text { Sustenta sus intervenciones } \\
\text { con argumentos sencillos. }\end{array}$ & $\begin{array}{l}\text { Da razones coherentes } \\
\text { dando a conocer las ideas } \\
\text { que tiene sobre el tema con } \\
\text { argumentos completos. }\end{array}$ \\
\hline Sacar conclusiones & $\begin{array}{l}\text { Sus conclusiones son } \\
\text { confusas y omite infor- } \\
\text { mación relevante. }\end{array}$ & $\begin{array}{l}\text { Plantea conclusiones sen- } \\
\text { cillas a partir de la informa- } \\
\text { ción recibida. }\end{array}$ & $\begin{array}{l}\text { Realiza con facilidad con- } \\
\text { clusiones claras que van } \\
\text { más allá de la información } \\
\text { recibida. }\end{array}$ \\
\hline
\end{tabular}

Fuente: elaboración propia.

Una vez planeadas y puestas en marcha las unidades de EpC, se evalúan los movimientos del pensamiento apoyándose en la matriz expuesta, con el fin de verificar el alcance de estos en los procesos de aprendizaje y enseñanza. Sin embargo, al observar el trabajo en el aula registrado en los diarios de campo y en las transcripciones de los videos de clase, se encuentra que los estudiantes tienen mayor inquietud y afinidad con los movimientos de pensamiento expuestos en la tabla 2, en la categoría de pensamiento, los cuales son: interpretar y explicar, razonar y argumentar y, por último, sacar conclusiones. Tabla 6. Matriz Movimientos del pensamiento

\section{Algunos resultados obtenidos}

En los niños se produjo un avance significativo en los procesos de comprensión, manejo de información a la que accedían y en la forma de comunicarse más clara y analítica. Al sistematizar y analizar la información recogida en los proyectos finales de síntesis de cada unidad de EpC, los cuales estaban relacionados con producciones escritas, se evidenció un verdadero avance en la adquisición de conocimiento y comprensión con el uso de las figuras literarias, la macro y microestructura de los textos y el uso adecuado de los conectores. En la figura 4 se muestra el avance significativo de unidad a unidad, teniendo en cuenta que la unidad uno se planeó y aplicó fuera de los criterios de EpC.

De igual forma, la docente notó un cambio positivo en su propio actuar al abrir los espacios para la discusión y la socialización y evidenciar que los niños pueden hacer más de que ella pensaba. Además, su propio lenguaje fue modificado, lo que le permitió ver desde otra perspectiva el aula de clase. 


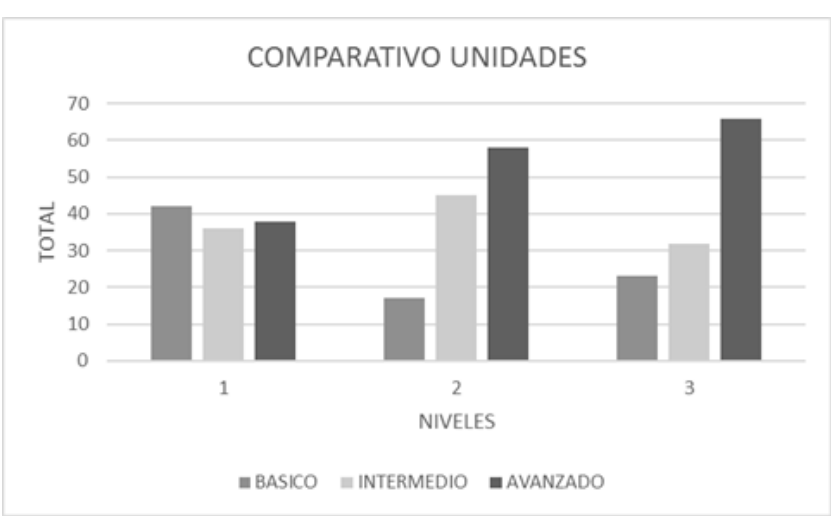

Figura 4. Comparativo unidades de trabajo.

Fuente: elaboración propia.

Ahora bien, si con las transformaciones en las prácticas docentes y los buenos resultados obtenidos en tan corto tiempo, además del recorrido que por sus edades ya tenían los estudiantes de ciclo tres, surgen nuevas inquietudes relacionadas con: ¿qué resultados tendríamos si desde el primer grado de escolaridad se trabajara con los niños bajo los parámetros de EpC y se potenciarán desde temprana edad las habilidades del pensamiento?, ¿cómo hacer que los niños más pequeños se familiaricen con el vocabulario del pensamiento y qué estrategias podría trabajar el docente? Para intentar dar respuestas a estas y otras tantas preguntas se solicita trabajar con el grado primero y aplicar los nuevos conocimientos pedagógicos; y registrar todo el proceso con el fin de continuar con el proceso investigativo en el aula.

\section{Conclusiones}

El docente debe tener la capacidad de detectar las buenas y malas prácticas pedagógicas, las cuales tienen un efecto positivo o negativo en los estudiantes que tiene a cargo. Una de esas prácticas negativas a modificar es el mantener paradigmas tradicionales; es decir, el ver a sus estudiantes como cubos a los que debe llenar de conceptos y temas que finalmente se van modificando con el pasar del tiempo. Es necesario hacer un alto y verificar si realmente se está preparado para educar en el pensamiento, el cual es la piedra angular de estas generaciones. Puesto que la información hoy en día ya está disponible, es necesario enseñar a utilizarla de forma adecuada y eso solo se hace con el pensamiento y el desarrollo de sus habilidades.

El uso constante del vocabulario del pensamiento permitió a los estudiantes tener conciencia de la importancia de este y de su adecuada utilización en los procesos comunicativos y en la apropiación del nuevo lenguaje del pensamiento. Pero, la transformación no se logró solo en los estudiantes, también la docente experimentó un cambio en sus propias prácticas pedagógicas y en el fortalecimiento de habilidades de pensamiento. Ello hizo que sus clases fueran más amenas y, mejor aún, más comprensibles y participativas, lo que provocó que los estudiantes realmente evidenciaran sus pensamientos.

Cuando se toma real conciencia sobre la importancia del trabajo docente y cómo este influye en las vidas de los estudiantes, incluso el de sus familias, se hace más responsable, indaga, se prepara y busca estrategias que permiten mejorar sus prácticas pedagógicas; como es el caso de la EpC, la cual admite realizar un verdadero acercamiento al aprendizaje comprensible y aplicable a diferentes contextos académicos, familiares, sociales y laborales. Como lo plantea Rodríguez: "El cambio educativo pasa por el cambio de las personas involucradas en los procesos de autorreflexión, fundamentalmente los docentes" (2005, p. 44).

Los niños tomaron real conciencia sobre la importancia de dar a conocer sus pensamientos por medio de la escritura, además de reconocer que siempre lo pueden hacer mejor al poner en práctica consiente las pautas necesarias para realizar un buen escrito, enriquecido con un buen vocabulario (producto de las lecturas realizadas en clase y de forma personal).

\section{Referencias}

Alvares, J. (2009). Aprender cómo aprendo: la enseñanza de estrategias metacognitivas. Educación y Educadores, 12(2), 11-28.

Cassany, D. (2007). Describir el escribir. Paidós. Guzmán, R. J., Verela, S. y Jorge, H. (2010). Referentes para la didáctica del lenguaje en el tercer ciclo. Kimpres. 
Johnson, P. A. (2003). El desarrollo de las habilidades del pensamiento. Troquel S.A.

Richhart, R., Church, M. y Morrison, K. (2014). Hacer visible el pensamiento. Paidós.

Rodríguez, J. S. (2005). La investigación acción ¿Qué es? ¿Cómo se hace? Doxa.

Romero, R. F., Restrepo, A. M. y Schwannflugel, P. (2009). Promoción del alfabetismo inicial y prevención de las dificultades en la lectura: una experiencia pedagógica en el aula de preescolar. Avances en Psicología Latinoamericana, 17, 79-96.
Ruiz, J. O. (2006). El cambio posible: educación centrada en el desarrollo del pensamiento. Revista Iberoamericana de Educación, 39(6).

Stone, M. (1999). La enseñanza para la comprensión. Paidós.

Thishman, S., Perkins, D. y Jay, E. (2006). Un aula para pensar. Aique.

Villarini, Á. R. (2003). Teoría y pedagogía del pensamiento crítico. Perspectiva Psicológica, 3-4(4), 35-42.

Vygotsky, L. S. (1995). Pensamiento y lenguaje. Fausto.

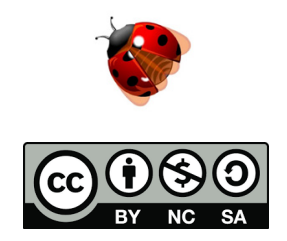

Esta obra está bajo licencia Creative Commons Atribución-NoComercial-Compartirlgual 4.0 Internacional 